\title{
Helical Twisting Power of Poly( $\gamma$-benzyl L-glutamate) Liquid Crystals in Mixed Solvents
}

\author{
Hirokazu ToriUmi, ${ }^{*}$ Sadaomi MinaKUCHI, ${ }^{*}$ Yoshiko UEMATSU, ${ }^{* *}$ \\ and Ichitaro UEMATSU* \\ * Department of Polymer Technology, Tokyo Institute of Technology., \\ 2-12-1 Ookayama, Meguro-ku, Tokyo 152, Japan. \\ **Faculty of Engineering, Tokyo Institute of Polytechnics, \\ Iiyama, Atsugi, Kanagawa 243-02, Japan.
}

(Received December 25, 1979)

\begin{abstract}
The helical twisting power of $\operatorname{poly}(\gamma$-benzyl L-glutamate $)$ liquid crystals in mixed solvents has been examined as a function of the solvent composition and temperature. Dioxane, ethylene dichloride, $m$-cresol, and trifluoroacetic acid have been employed as solvents. The helical twisting power in the dioxane-ethylene dichloride system varies linearly with the solvent composition, while in the $m$-cresol-ethylene dichloride system, it varies as a quadratic function of the solvent composition. The addition of a small amount of trifluoroacetic acid (up to $6 \%$ ) to the ethylene dichloride solution causes an increase in the helical twisting power. The solvent effect of $m$ cresol and trifluoroacetic acid has been discussed in terms of the intermolecular hydrogen-bond formation to the side-chain carbonyl ester groups and the disruption of aggregates of poly $(\gamma$-benzyl L-glutamate) $\alpha$-helices.
\end{abstract}

KEY WORDS Poly( $\gamma$-benzyl L-glutamate)/Liquid Crystal / Mixed Solvent /

Helical Twisting Power / Cholesteric Sense / Inversion / Solvent Effect /

Hydrogen Bond /

The cholesteric liquid-crystalline structure can be characterized by the cholesteric sense (right-handed or left-handed) and the cholesteric pitch. The pitch $P$ is related to the twisting angle $\phi$ between neighboring molecules separated by a distance $d$ along the axis of torsion as follows,

$$
\phi=2 \pi d / P .
$$

Thus, the reciprocal value of $P$ provides a measure of the helical twisting power.

In thermotropic liquid crystals, the cholesteric sense is determined by the chirality of the constituent molecule; the cholesteric sense of the liquid crystal of an optical isomer must be opposite to that of its mirror image isomer. When equal moles of both isomers are mixed (racemic mixture), the twisting power falls to zero and the cholesteric structure changes to a compensated nematic structure. Such compensation phenomena have been known for many binary mixtures of cholesteric materials or those of cholesteric and nematic materials. For most of these systems, the twisting power has been found to vary linearly with the content of one component. ${ }^{1,2}$ However, there are some examples in which the twisting power changes in a non-linear fashion with the composition. ${ }^{3-8}$ In order to analyze the experimental results, Finkelmann and Stegemeyer ${ }^{5,6}$ have applied the molecular theory of Goossens ${ }^{9}$ to the binary systems. Their theory predicts a quadratic dependence of the twisting power on the composition.

The compensation phenomena have been also known for lyotropic polypeptide liquid crystals in organic solvents. A racemic mixture of $\operatorname{poly}(\gamma-$ benzyl L-glutamate) (PBLG, a right-handed $\alpha$-helix) and its mirror image isomer PBDG (a left-handed $\alpha$ helix) forms the nematic liquid crystal. ${ }^{10,11}$ However, a more surprising observation is that the cholesteric sense of the PBLG liquid crystal depends on the nature of the solvent. For example, PBLG liquid crystals in chloroform and in dioxane form the right-handed cholesteric structure, while in 
methylene chloride and in ethylene dichloride (EDC), form the left-handed one. In an appropriate solvent mixture of dioxane and methylene chloride (at $20 \mathrm{vol} \%$ of dioxane), the cholesteric structure changes to the compensated nematic structure. Recently, we ${ }^{12}$ reported a new type of compensation induced by a change in temperature. The PBLG liquid crystal in $m$-cresol forms the right-handed cholesteric structure at low temperatures, but it undergoes an inversion to the left-handed cholesteric structure around $60^{\circ} \mathrm{C}$. A similar phenomenon was also observed in the benzyl alcohol solution.

In this study, we have employed dioxane, EDC, and $m$-cresol as solvents, and have determined the helical twisting power of PBLG liquid crystals in mixtures of solvents mentioned above. The observed dependence of the twisting power on the solvent composition has been analyzed based on the theory of Finkelmann and Stegemeyer. In order to examine the origin of the solvent effect, we have further examined the influence of trifluoroacetic acid (TFA) on the twisting power of the PBLG-EDC liquid crystal. TFA is known as an agent which breaks the aggregates of PBLG $\alpha$-helices.

\section{EXPERIMENTAL}

The sample of PBLG was synthesized by the polymerization of $N$-carboxyanhydride of $\gamma$-benzyl L-glutamate using triethylamine as the initiator in dioxane. The average molecular weight estimated by the viscosity measurement was about $18.4 \times 10^{4}$. Dioxane, EDC, and $m$-cresol were used as solvents following vacuum-distillation. TFA (guaranteed reagent grade) purchased from Tokyo Kasei Co., Ltd. was used without further purification. A solution containing a $15 \%$ volume fraction of PBLG was sealed in a glass cell of $2 \mathrm{~mm}$ thickness, and then was matured at room temperature to allow for the formation of the liquid-crystalline structure. The cholesteric half-pitch $S(=P / 2)$ was determined from observations of the spacings between the retardation lines under a polarizing microscope. The pitch measurements were carried out at every $5^{\circ} \mathrm{C}$ intervals in a thermostated oven which kept the temperature fluctuation less than $\pm 0.1^{\circ} \mathrm{C}$. The infrared (IR) spectra were measured by a JASCO DS-701G Infrared Spectrophotometer using a KRS-5 (thallium bromide-thallium iodide mixed crystal) cell.

\section{RESULTS}

\section{Helical Twisting Power of PBLG Liquid Crystals in Mixed Solvents}

Figure 1 shows the temperature dependence of the reciprocal value of cholesteric half-pitch $\left(S^{-1}\right)$ of PBLG liquid crystals in dioxane-EDC mixed solvents. The sign of the $S^{-1}$ value is taken as positive and negative for the right- and left-handed cholesteric structures, respectively. In dioxane, the right-handed cholesteric structure is formed and its twisting power decreases when temperature is increased. On the other hand, PBLG in EDC forms the left-handed cholesteric structure where the lefthanded twisting power increases with temperature. Thus, the temperature coefficient of the twisting power is always negative for both systems. The addition of EDC to the dioxane solution causes a reduction of the right-handed twisting power, and produces a downward shift of the $S^{-1} v s$. temperature curve. In a mixture of $40 \mathrm{vol} \%$ dioxane and $60 \mathrm{vol} \%$ EDC, the liquid crystal undergoes a thermally-induced sense inversion from right to left, at about $47^{\circ} \mathrm{C}$. In all of these systems, a linear relationship between the twisting power and temperature holds.

In Figure 2, the twisting power is plotted against the solvent composition in the dioxane-EDC mixed solvent system at various temperatures. It is clear

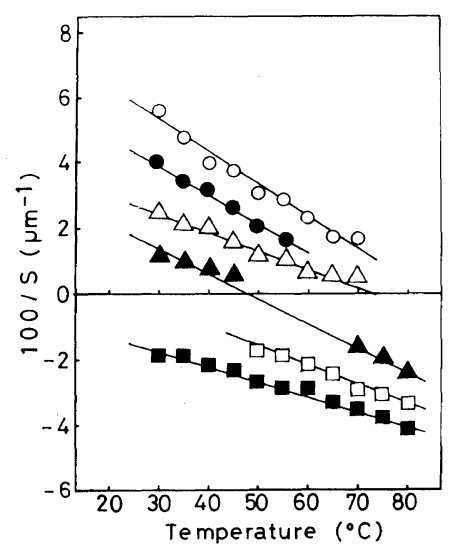

Figure 1. Temperature dependence of the helical twisting power of PBLG liquid crystals in dioxane-EDC mixed solvents. The contents (vol \%) of EDC in solvent mixtures: $(\bigcirc) 0,(\bigcirc) 20,(\triangle) 40,(\boldsymbol{\Delta}) 60,(\square) 80$, and ( 100. 


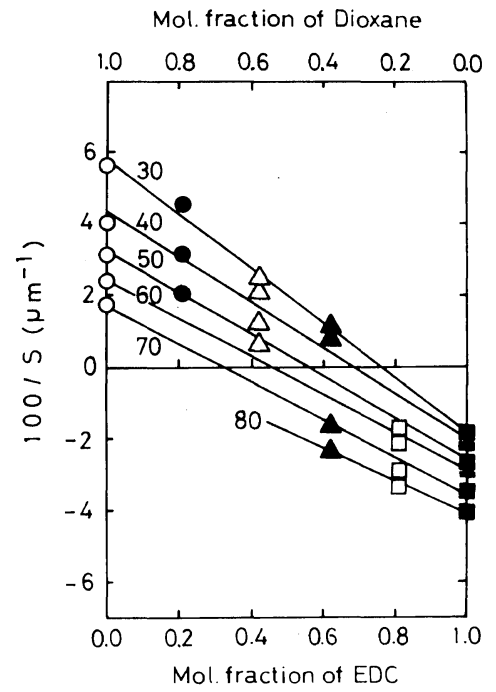

Figure 2. Dependence of the helical twisting power on the solvent composition in dioxane-EDC mixtures. Numerals indicate the temperatures in ${ }^{\circ} \mathrm{C}$.

that a linear additive law for the twisting power is achieved at each temperature. The critical composition of the solvent at which compensation occurs shifts to the higher dioxane content region as the temperature is increased.

Figures $3 \mathrm{a}$ and $3 \mathrm{~b}$ show the temperature dependence of the twisting power in the $m$-cresolEDC system. The cholesteric sense of the PBLG liquid crystal in $m$-cresol inverts from right to left as the temperature is raised above $60^{\circ} \mathrm{C}$. The addition of EDC to this system causes a shift of the compensation temperature to $37^{\circ} \mathrm{C}$ at $20 \mathrm{vol} \% \mathrm{EDC}$ and to below $20^{\circ} \mathrm{C}$ at $40 \mathrm{vol}^{\circ} \%$ EDC. At the same time, the temperature coefficient of the twisting power gradually decreases. The downward shift of the $S^{-1} v s$. temperature curve continues up to $60 \mathrm{vol}^{\%} \%$ EDC (Figure 3a), but a further addition of EDC causes an upward shift (Figure $3 b$ ). This suggests a quadratic dependence of the twisting power on the solvent composition.

This can be clearly recognized in Figure 4, where the twisting power is plotted against the solvent composition. When the EDC content is increased at low temperatures, the right-handed twisting power rapidly decreases to zero and inverts to the lefthanded twisting power. In the higher EDC content region, the left-handed twisting power begins
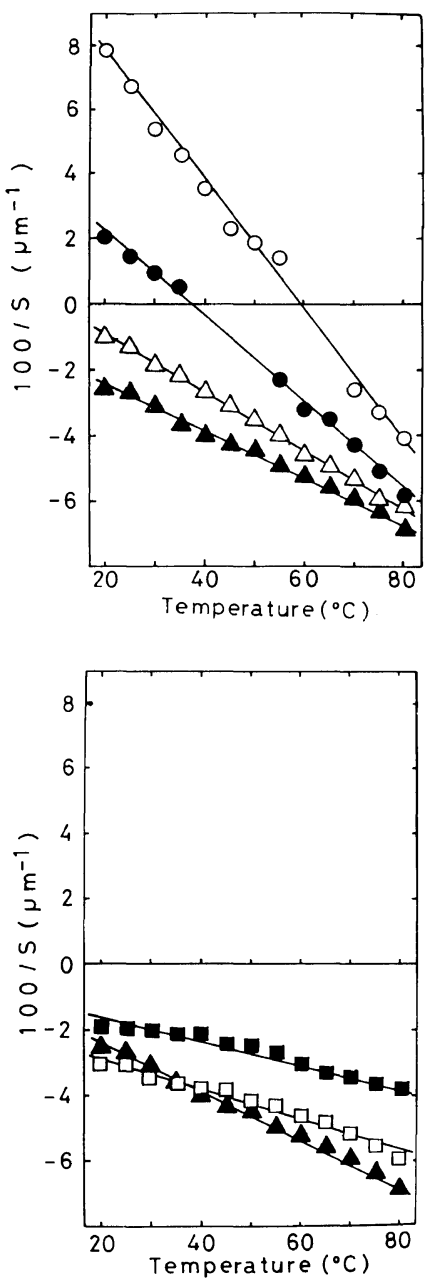

Figure 3. Temperature dependence of the helical twisting power of PBLG liquid crystals in $m$-cresol-EDC mixed solvents. The contents ( $\mathrm{vol} \%$ ) of EDC in solvent

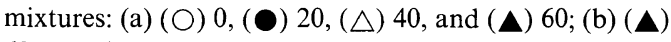
$60,(\square) 80$, and (口) 100 .

decreasing after going beyond an extreme value. The deviation of the experimental curve from the linear additive law seems largest at about 50\% EDC. The solvent-induced compensation disappears above $60^{\circ} \mathrm{C}$, but the twisting power still maintains its quadratic dependence on the solvent composition.

More complicated behavior was observed in the $m$-cresol-dioxane system. The twisting power $v s$. the solvent composition curve in Figure 5 shows both the maximum and minimum peaks at low tempera- 


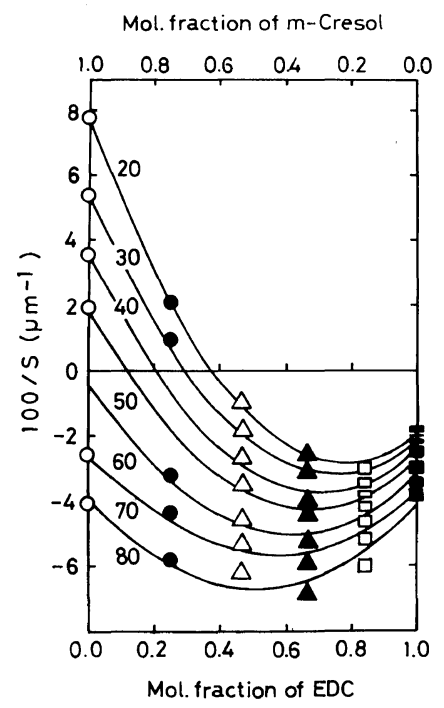

Figure 4. Dependence of the helical twisting power on the solvent composition in $m$-cresol-EDC mixtures. Numerals indicate the temperatures in ${ }^{\circ} \mathrm{C}$.

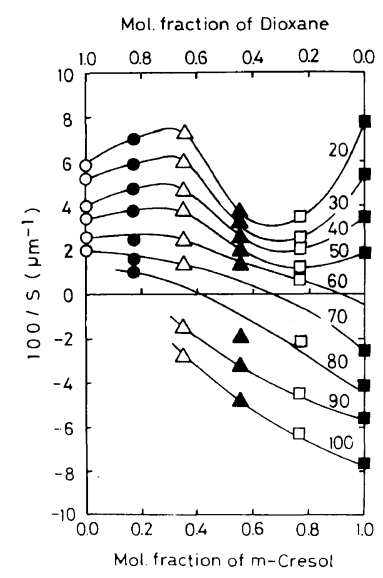

Figure 5. Dependence of the helical twisting power on the solvent composition in $m$-cresol-dioxane mixtures. Numerals indicate the temperatures in ${ }^{\circ} \mathrm{C}$.

tures. The initial increase in the right-handed twisting power caused by the addition of $m$-cresol might arise from a similar mechanism bringing about the increase in the left-handed twisting power of the PBLG-EDC liquid crystal. The origin of the minimum peak around $70 \% \mathrm{~m}$-cresol is not clear at this stage. At higher temperatures where the cholesteric structure in $m$-cresol changes to the left- handed structure, the minimum peak disappears and the curve becomes rather monotonous.

\section{Effect of TFA on the Liquid-Crystalline Structure in the PBLG-EDC System}

It is known that $\alpha$-helices of PBLG aggregate in certain solvents and that the structure of this aggregate depends on the solvent. ${ }^{13-15}$ In the EDC solution, a linear head-to-tail type aggregation has been suggested for PBLG. ${ }^{16,17}$ The addition of small amounts of certain agents (dimethylformamide, dichloroacetic acid, and TFA) to the system causes a disruption of the aggregated PBLG helices, as indicated by a decrease in the viscosity or by a change in the dipole moment. ${ }^{13,14,18}$

When few percents of TFA (up to $6 \%$ ) were added to the PBLG-EDC liquid crystal, the fluidity of the solution was increased markedly. This behavior is similar to that in dilute solutions, thus indicating the breakdown of the aggregates. A further addition of TFA $(8 \%)$ results in the phase separation of a small portion of an isotropic phase from the liquidcrystalline phase. According to the Flory theory of the phase separation, ${ }^{19,20}$ a critical concentration of polymer necessary to form the liquid-crystalline phase is expected to shift to higher values when the axial ratio (length-to-width ratio) of polymer is decreased or the flexibility is increased. Thus, this observation can be ascribed to the disruption of the linear head-to-tail type aggregation and/or to the increase in the flexibility of PBLG $\alpha$-helices. ${ }^{21}$ The effects of TFA on the helical twisting power are shown in Figures 6 and 7. The left-handed twisting

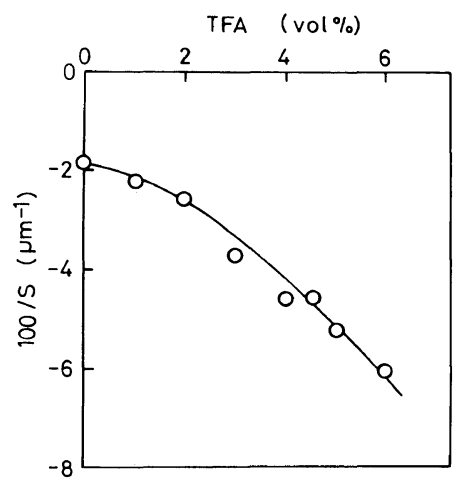

Figure 6. Dependence of the helical twisting power of the PBLG liquid crystal on the TFA content in EDCTFA mixtures at $30^{\circ} \mathrm{C}$. 


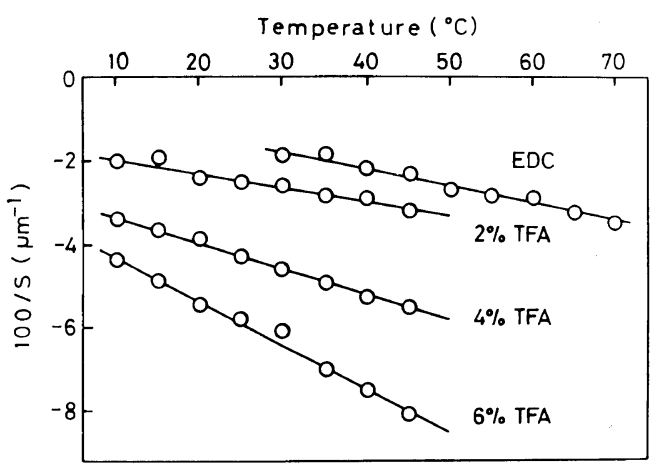

Figure 7. Temperature dependence of the helical twisting power of PBLG liquid crystals in EDC-TFA mixtures at various TFA concentrations.

power gradually increases with the TFA content. At the same time, the temperature coefficient of the twisting power increases. A similar observation was reported for the PBLG-dioxane liquid crystal, wherein the right-handed twisting power increases with TFA content. ${ }^{22}$ In both systems, disruption of the aggregates is accompanied by an increase in the twisting power of the starting liquid crystal, regardless of the cholesteric sense.

\section{DISCUSSION}

Finkelmann and Stegemeyer ${ }^{5,6}$ have analyzed the composition dependence of the twisting power in binary mixtures of thermotropic liquid crystals, using the Goossens theory. ${ }^{9}$ The twisting angle $\phi$ between the directors of two nematic layers is given as follows for a mixture of molecules of types 1 and 2 ,

$\phi=\frac{1}{d} \frac{B_{11} x^{2}+\left(B_{12}+B_{21}\right) x(1-x)+B_{22}(1-x)^{2}}{A_{11} x^{2}+2 A_{12} x(1-x)+A_{22}(1-x)^{2}}$

where $d$ is the distance between two directors and $x$ is the molar fraction of component 1 . The parameter $A_{i j}$ is related to the anisotropy of the molecular polarizability, and $B_{i j}$ is related to the dispersion energy determined by dipole-quadrupole interactions. A non-vanishing $B_{i j}$ value, responsible for the cholesteric twist, is attained only for optically active molecules. In the case of binary mixtures of cholesteric molecules having chemically similar properties, one would expect $A_{11} \simeq A_{22} \simeq A_{12}=A .^{7,8}$ Then, by substituting $\phi_{i j}=B_{i j} / d A$, eq 2 can be reduced to,

$$
\phi=\phi_{11} x^{2}+\left(\phi_{12}+\phi_{21}\right) x(1-x)+\phi_{22}(1-x)^{2}
$$

Further, by using the relationship $\phi=\pi d / S$, we get

$$
S^{-1}=S_{11}^{-1} x^{2}+\left(S_{12}^{-1}+S_{21}^{-1}\right) x(1-x)+S_{22}^{-1}(1-x)^{2}
$$

Thus, the twisting power in binary mixtures is characterized by the quadratic dependence on the composition and by the appearance of the cross terms $S_{12}^{-1}$ and $S_{21}^{-1}$ representing the induced twisting powers. If molecules 1 and 2 in a mixture satisfy the conditions $B_{21} \simeq B_{11}$ and $B_{12} \simeq B_{22}$. eq 2 may be further reduced to,

$$
S^{-1}=S_{11}^{-1} x+S_{22}^{-1}(1-x)
$$

This equation provides the twisting power which varies linearly with composition. When $S_{11}^{-1}$ and $S_{22}^{-1}$ have mutually opposite signs, the inversion of the cholesteric sense should occur at a critical composition, $x^{*}=S_{22}^{-1} /\left(S_{22}^{-1}-S_{11}^{-1}\right)$.

The Goossens theory is based on the assumption that the cholesteric molecules are planar and lie in layers, stacked perpendicularly to the axis of torsion. As a consequence of this theory, it is suggested that cylindrically symmetric molecules do not form the cholesteric twist. This suggestion, however, does not hold true for PBLG liquid crystals. The PBLG molecule takes on the $\alpha$-helical conformation and behaves as a rod-like molecule. The supramolecular structure in the liquidcrystalline solution of PBLG is locally uniaxial and is regarded to have a twisted nematic (chiral nematic) structure. In spite of this, the dependence of the twisting power of PBLG liquid crystals on the solvent composition is well described by the extended Goossens theory, provided we use $x$ as the molar fraction of one solvent component in solvent mixture. The twisting power in the dioxane-EDC system varies linearly with the solvent composition, and thus it can be fitted to eq 5. On the other hand, in the $m$-cresol-EDC system, this twisting power has a quadratic nature, as indicated by eq 4 . The solid curves in Figures 2 and 4 show the least square fits of the data, using $S_{i j}^{-1}$ as fitting parameter. The values of $S_{i j}^{-1}$ thus obtained are summarized in Table I. Experimentally, $S_{12}^{-1}$ and $S_{21}^{-1}$ cannot be distinguished, so we use $\left(S_{12}^{-1}+S_{21}^{-1}\right) / 2$ instead. Table I also includes the critical solvent composition $x^{*}$.

The substitution of dioxane by $m$-cresol results in 
Table I. Temperature dependence of the helical twisting power $S_{i j}^{-1}$ and the critical solvent composition for compensation $x^{*}$

\begin{tabular}{|c|c|c|c|c|c|c|}
\hline \multirow{2}{*}{ Solvent 1} & \multirow{2}{*}{ Solvent 2} & \multirow{2}{*}{$\frac{\text { Temp }}{{ }^{\circ} \mathrm{C}}$} & \multirow{2}{*}{$\frac{S_{11}^{-1}}{\mu \mathrm{m}^{-1}}$} & \multirow{2}{*}{$\frac{S_{22}^{-1}}{\mu \mathrm{m}^{-1}}$} & \multirow{2}{*}{$\frac{\left(S_{12}^{-1}+S_{21}^{-1}\right) / 2}{\mu \mathrm{m}^{-1}}$} & \multirow{2}{*}{$x^{* \mathrm{a}}$} \\
\hline & & & & & & \\
\hline \multirow{6}{*}{ Dioxane } & \multirow{6}{*}{ EDC } & 30 & 0.058 & -0.019 & & 0.24 \\
\hline & & 40 & 0.044 & -0.020 & & 0.32 \\
\hline & & 50 & 0.033 & -0.026 & & 0.44 \\
\hline & & 60 & 0.024 & -0.029 & & 0.54 \\
\hline & & 70 & 0.017 & -0.036 & & 0.68 \\
\hline & & 80 & 0.006 & -0.040 & & 0.88 \\
\hline \multirow{7}{*}{$m$-Cresol } & \multirow{7}{*}{$\mathrm{EDC}$} & 20 & 0.078 & -0.020 & -0.029 & 0.62 \\
\hline & & 30 & 0.055 & -0.023 & -0.030 & 0.70 \\
\hline & & 40 & 0.037 & -0.024 & -0.035 & 0.80 \\
\hline & & 50 & 0.020 & -0.027 & -0.037 & 0.88 \\
\hline & & 60 & -0.004 & -0.032 & -0.040 & \\
\hline & & 70 & -0.024 & -0.037 & -0.041 & \\
\hline & & 80 & -0.039 & -0.041 & -0.047 & \\
\hline
\end{tabular}

a Molar fraction of solvent 1 in solvent mixture.

a remarkable influence on the twisting power. The deviation from the linear additive law observed in the $m$-cresol-EDC system indicates that the effect of adding $m$-cresol increases the left-handed twisting power of the PBLG-EDC liquid crystal. Similarly, by adding $m$-cresol to the dioxane solution, the right-handed twisting power increases (Figure 5). It is interesting to compare these observations with the results in the EDC-TFA mixed solvent; a trace amount of TFA increases the left-handed twisting power of the PBLG-EDC liquid crystal. In dilute solutions, it is known that halogenated acids interact with PBLG molecules by hydrogen-bond formation with the side-chain carbonyl ester groups ${ }^{23}$ or by protonation of the terminal amide groups of the main chain. ${ }^{24}$ The disruption of PBLG aggregates is considered to occur in a consequence of such specific polymer-solvent interactions. ${ }^{13}$ Figure 8 shows the IR spectra of the carbonyl ester groups of PBLG in liquid-crystalline solutions in EDC, dioxane, and $m$-cresol. The wavenumbers of maximum absorbance are $1735 \mathrm{~cm}^{-1}$ in EDC and dioxane, but $1715 \mathrm{~cm}^{-1}$ in $m$-cresol. Peaks at 1735 and $1715 \mathrm{~cm}^{-1}$ have been assigned to the carbonyl groups in the free and in the hydrogen-bonded state, respectively. ${ }^{12,25} \mathrm{It}$ appears that the carbonyl groups in the $m$-cresol solution are hydrogen-bonded with solvent mo- lecules, thereby differing from those in the other two solvents. Disaggregation phenomena in dilute solutions suggest that $m$-cresol added to the EDC or dioxane solution, breaks down the aggregates of the

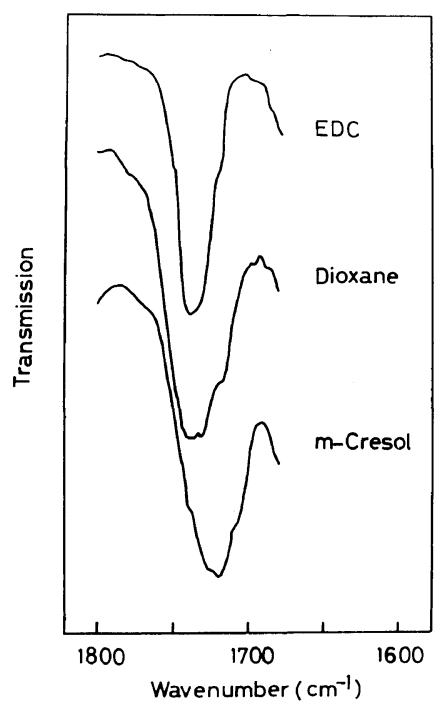

Figure 8. The IR absorption spectra of the side-chain carbonyl ester groups of PBLG in liquid-crystalline solutions in EDC, dioxane and $m$-cresol. 
PBLG $\alpha$-helices. The initial increases in the twisting power caused by $m$-cresol or TFA may then be attributed to the disruption of the aggregates.

Recently, Samulski and Samulski ${ }^{26}$ have presented a discussion on the solvent effect in terms of relative magnitudes of the dielectric constants of PBLG and solvent molecules. This theory predicts that compensation occurs for a critical value of the dielectric constant of solvent $\varepsilon^{*}=\left(\varepsilon_{1} \varepsilon_{2}\right)^{1 / 2}$, where $\varepsilon_{1}$ and $\varepsilon_{2}$ are the principal values of the dielectric constant perpendicular to the long molecular axis of PBLG. This indicates that the twisting power changes its sign when the dielectric constant of the solvent is varied on passing through $\varepsilon^{*}$ by the addition of second solvent component. This theory seems to be true for the inversion phenomena in the dioxaneEDC system, where the right-handed cholesteric structure in dioxane $(\varepsilon=2.2)$ changes to the lefthanded structure in EDC $(\varepsilon=10.5)$. With regard to the dielectric constant, one would expect $m$-cresol $(\varepsilon=11.8)$ to behave as EDC, i.e., $m$-cresol supports the left-handed cholesteric structure. However, the aforementioned study shows that $m$-cresol supports the right-handed structure at low temperatures. This discrepancy may again be ascribed to the shortrange interactions of $m$-cresol with the PBLG side chains. Milstien and $\mathrm{Charney}^{23}$ have noted that the formation of intermolecular hydrogen bonds changes the orientation of permanent dipole moments located on the carbonyl ester groups. This may be seen by a reduction of the overall dipole moment of the PBLG molecule with the addition of TFA. If such a change in the side-chain environment alters the anisotropy in the dielectric constants of PBLG $\left(\varepsilon_{1}\right.$ and $\left.\varepsilon_{2}\right)$, then in accordance with the theory of Samulski and Samulski, we may expect the magnitude and sign of the twisting power in the $m$-cresol solution to be affected. It seems that the formation of the right-handed cholesteric structure in benzyl alcohol $^{12}(\varepsilon=11.0)$ may also be explained by this mechanism.

\section{REFERENCES}

1. H. Baessler and M. M. Labes, J. Chem. Phys., 52, 631 (1970).

2. D. Dolphin, Z. Muljiani, J. Cheng, and R. B. Meyer, J. Chem. Phys., 58, 413 (1973).

3. J. E. Adams and W. E. Hass, Mol. Cryst. Liq. Cryst., 15, 27 (1971).

4. T. Nakagiri, H. Kodama, and K. K. Kobayashi, Phys. Rev. Lett., 27, 564 (1971).

5. H. Stegemeyer and H. Finkelmann, Chem. Phys. Lett., 23, 227 (1973).

6. H. Finkelmann and H. Stegemeyer, Ber. Bunsenges. Phys. Chem., 78, 869 (1974).

7. H. Hanson, A. J. Dekker, and F. van der Woude, $J$. Chem. Phys., 62, 1941 (1975).

8. H. Hanson, A. J. Dekker, and F. van der Woude, Mol. Cryst. Liq. Cryst., 42, 15 (1977).

9. W. J. A. Goossens, Mol. Cryst. Liq. Cryst., 12, 237 (1970).

10. C. Robinson, J. C. Ward, and R. B. Beevers, Discuss. Faraday Soc., 25, 29 (1958).

11. C. Robinson, Tetrahedron, 13, 219 (1961).

12. H. Toriumi, Y. Kusumi, I. Uematsu, and Y. Uematsu, Polym. J., 11, 863 (1979).

13. P. Doty, J. H. Bradbury, A. M. Holtzer, J. Am. Chem. Soc., 78, 947 (1956).

14. A. K. Gupta, C. Dufour, and E. Marchal, Biopolymers, 13, 1293 (1974).

15. J. C. Powers and W. L. Peticolas, Biopolymers, 9, 195 (1970).

16. H. Watanabe, Nippon Kagaku Zasshi, 86, 179 (1965).

17. H. Kihara, Polym. J., 7, 406 (1977).

18. A. Wada, J. Polym. Sci., 45, 145 (1960).

19. P. J. Flory, Proc. R. Soc., London, Ser. A, 243, 60 (1956).

20. P. J. Flory, Proc. R. Soc., London, Ser. A, 243, 73 (1956).

21. P. Laszlo, A. Paris, and E. Marchal, J. Phys. Chem., 77, 2925 (1973).

22. D. B. DuPré, R. W. Duke, W. A. Hines, and E. T. Samulski, Mol. Cryst. Liq. Cryst., 40, 247 (1977).

23. J. B. Milstien and E. Charney, Biopolymers, 9, 991 (1970).

24. H. Watanabe, K. Yoshioka, and A. Wada, Biopolymers, 2, 91 (1964).

25. E. M. Bradbury, A. R. Downie, A. Elliott, and W. E. Hanby, Proc. R. Soc., London, Ser. A, 259, 110 (1960).

26. T. V. Samulski and E. T. Samulski, J. Chem. Phys., 67, 824 (1977). 